\title{
Hybrid Fiber Radio Systems: Features and Performance
}

\author{
Roberto Sabella \\ Ericsson Telecomunicazioni, $R \& D-G P C$, Roma, Italy; E-mail: \\ roberto.sabella@tei.ericsson.se; fax +396 72597439; tel. +39 6 72597442; Via An \\ agnina 203, 00040, Rome, Italy.
}

Key words: Fiber-to-the Area Systems (FTTA), Hybrid Fiber-Radio Systems, Sub-Carrier Multiplexing, Access Network Architecture.

\begin{abstract}
The paper summarizes basic system design issues for an optical fiber feeder in either mobile radio or fixed radio access systems. The features of the introduction of the optical feeder are discussed, and the performance evaluated in some relevant cases, are reported.
\end{abstract}

\section{INTRODUCTION}

The low loss and large band features of the optical fibers have led to the realization and the installation of a variety of advanced communications systems throughout the world. At the same time, mobile communication systems, which allow people to communicate at anytime, anyplace and with anybody, are more and more diffused. Furthermore, the radio resource is going to be even more exploited to distribute broadband services to both business and residential customers, by using millimeter wave band. Fixed radio access systems are representing a valid alternative to realize access systems without the need of costly cable infrastructures.

The combination of fiber optic and radio technologies allows the realization of hybrid fiber radio (HFR) systems (even known as FTTA: fiber-to-the-antenna), which have an important advantage: easy and flexible connection of cells/microcells and, particularly, greatly simplified radio base 
stations. In addition, these systems can be upgraded very quickly, because there is no need to replace existing base station equipment.

Research in this field started several years ago (see as an example the whole special issue [1]), focused on microcellular systems. In fact, it is well known that microcellular systems, composed of small size cells with several hundred meter diameter communication range, promise effective frequency spectrum utilization. However, these systems require a large number of base stations. Therefore, especially for these systems, miniaturization of base stations is an important issue in realizing microcellular systems.

The HFR approach allows the miniaturization and a significant simplification of the base station architecture. Section 2 examines the HFR approach in a mobile case, while section 3 considers the FTTA case for wireless broadband systems. Finally, section 4 reports a discussion about the performance of these systems, and some general conclusions.

\section{HFR SYSTEM FOR MOBILE COMMUNICATIONS}

The hybrid approach was originally introduced for being applied in microcellular communication system [2]-[4].

In such systems, service areas are divided into many zones named "microcell" having radius of some hundred meters. In fact, since the base station's antennas are located about 10-15 $\mathrm{m}$ above the ground, they can only cover small area. This system approach allows frequency reuse, and can accommodate a large numbers of subscribers. On the other hand, this system has the disadvantages that a large number of base stations (BS) are required because of the large number of microcells required to cover a service area. Furthermore, personal stations frequently move across microcells, so channel assignment and hand-off procedure is much complicated.

To solve this problem, it was proposed to connect microcell BS with a microcell control station (CS) using an optical fiber link, in which the signal obtained by frequency division multiplexing of the telephone channels directly modulates a laser source (SCM: sub-carrier multiplexing) [2]-[4]. In this kind of system, the BS only converts the radio frequency signal to an optical signal: the required equipment for BS is remarkably simplified compared with the conventional system. The BS simplicity is an important feature of the system, because many BS would be typically required and, therefore, they should be economical and trouble-free in remote locations. Furthermore, channel assignment and hand-off procedure are centralized. The layout of the microcellular system is identical to the existing cellular radio one except fibers and base stations have been inserted between the mobile units and the central stations. 
Intensity modulated optical signal is transmitted over optical fiber to microcell CS, where optical signal is directly detected using a p-i-n photodiode, and a bandpass filter is used to provide RF channel signal. After filtering, each RF signal is demodulated and connected to the public switched telephone network. The system configuration is schematically reported in figure 1.

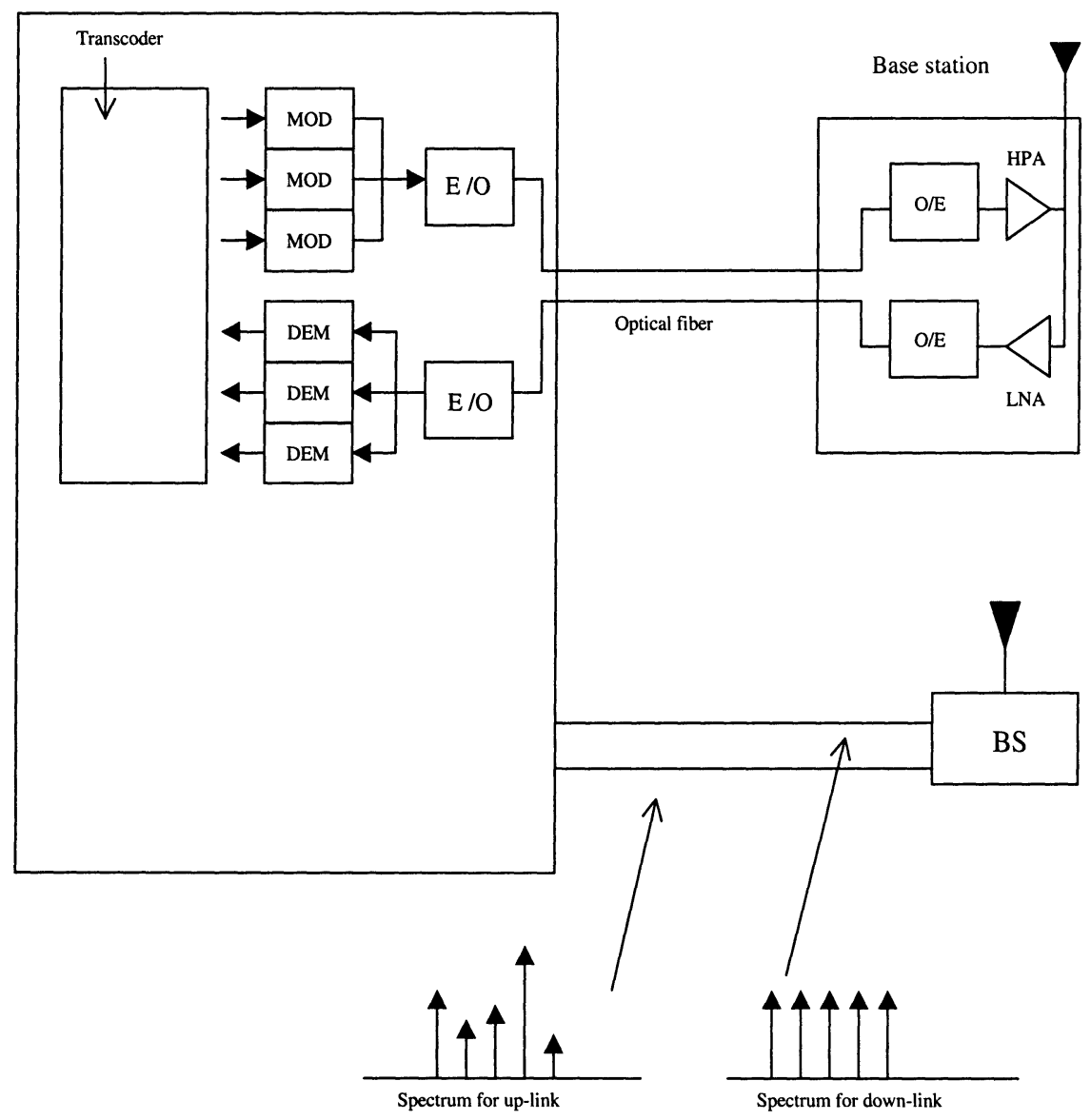

Figure 1. Basic configuration of FTTA system

For mobile radio applications, the optical feeder is required to have low noise and low-distortion characteristics. This requirement is particularly stringent for the up link feeder, since the radio signal received at the BS fluctuates greatly due to fading, shadowing and changes in distance between the micro BS and the mobile stations.

Although laser diode characteristics in analog modulation have been improved with respect to the past (e.g. in 90s), the feeder dynamic range is 
still limited by laser diode noise and non-linearity. So, using SCM technique, optical source non-linearity must be taken into account. In fact the non-linear characteristic of the laser diode causes several inter-channel interference, especially when many sub-carriers are transmitted from a single source, that is, for high total modulation indices. Furthermore, even if the laser characteristic was linear above its threshold current, signal distortion occurs also because the laser intensity is occasionally clipped by the large negative excursions of the modulating current. This phenomenon called clipping, gives rise to impulsive noise that deteriorates the system performance [5]. Optical source non-linearity is sketched in figure 2 .

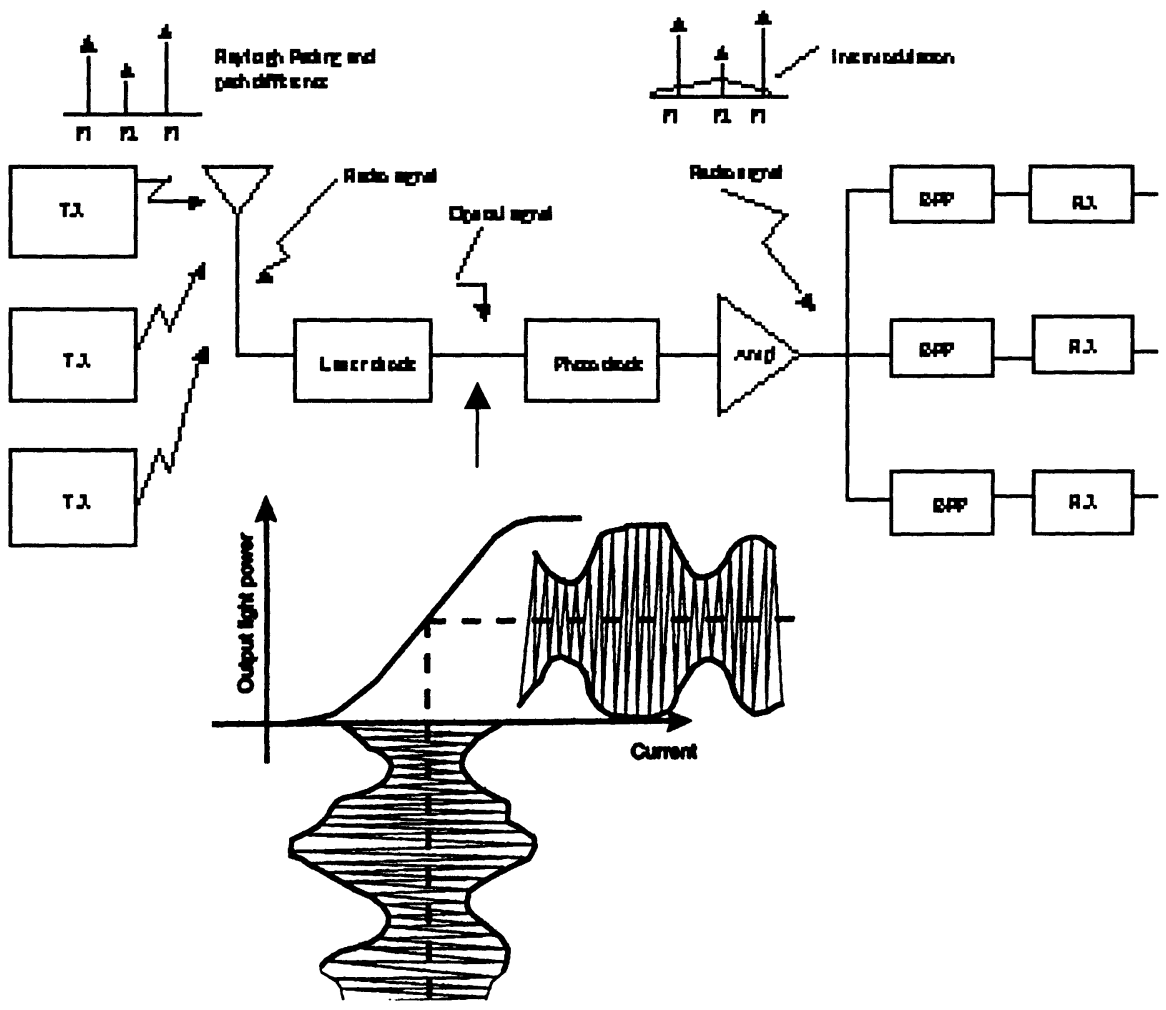

Figure 2. System degradation due to third-order intermodulation terms and clipping noise

The model that allows the evaluation of the transmission performance of the systems taking into account all these effects was reported in [6]. 


\section{FTTA SYSTEM FOR BROADBAND COMMUNICATIONS}

The employment of the optical feeder in broadband wireless systems is a very attractive option.

Before the description of the FTTA scheme considered in this paper it is worth while briefly mentioning the enabling technologies. The fiber optic technologies for microwave and millimeter-wave transmission are reviewed in [7], as well as different link architecture for RF signal transmission (i.e. directly modulation of the laser, external modulation, etc.). Furthermore, that paper discussed MMIC techniques for optical receivers, and stated that HEMT and HBT devices are very attractive in the realization of compact and cost effective optical receivers.

The enabling technologies were also discussed in a more recent paper [8]. In particular, the latter paper discussed three different approaches for linking the optical feeder with the radio link:

1. Baseband transport between the CS and the BS. This is the state of the art; in this case commercial equipment's can be used. However, when using this concept, the BS concentrates all the equipment's that depends on the physical medium of the final drop (e.g. coding, error correction, modulation and upconversion to IF, upconversion to RF, filtering and amplification).

2. Intermediate frequency (IF) transport. It makes possible to use BSs with fewer functions. This approach requires an optical IF transceiver, up/down conversion between the intermediate and radio frequencies, power amplification on the downlink, and a low noise front end on the uplink. All other functions, like line termination of the digital segment in the core network, mux/demux functions and router to map partial data streams to radio channels, access management for the shared radio medium, modems for signal adaptation to the radio channel, and up/down conversion to the IF, are performed in the CS.

3. Optical mm-wave RF transport. This is an advanced and attractive solution. Here the BS consists of an optical RF transceiver followed by a stage providing power amplification on the downlink and low noise amplification on the uplink. Promising techniques for the practical realization of mm-transport are based on a self heterodyning concept [9], and on the external modulation of the laser source by means of electro-optical modulators working at mm-waves [10].

In this paper we basically refer to the second approach (IF transport), which is synthetically depicted in fig. 3 [11]. 


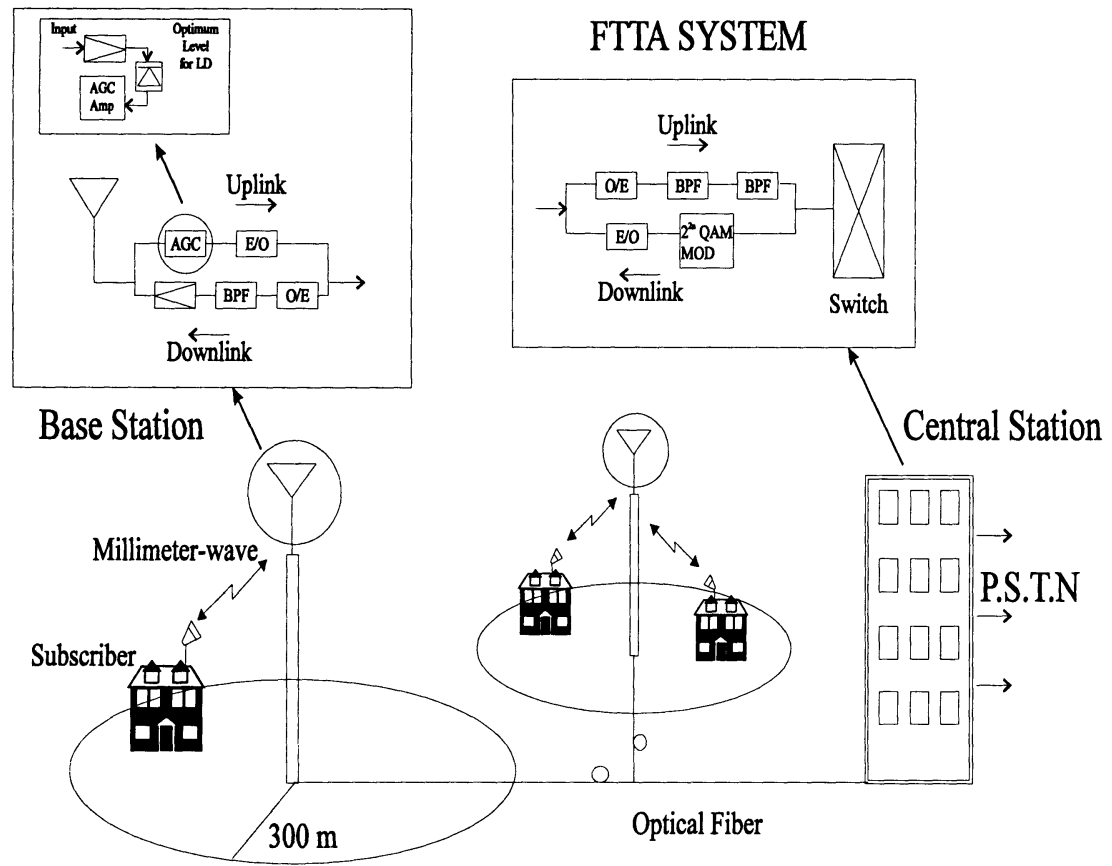

Figure 3. The concept of a FTTA system. P.S.T.N.:Public Switched Telecomunication Network; O/E or E/O: optoelectronic or electro/optic converter; BPF: bandpass filter; MOD: modulator;DEM: demodulator; AGC: automatic gain control circuit

In this system each zone radius can be of the order of hundred meters (between $300 \mathrm{~m}$ and $1 \mathrm{~km}$ in the considered cases). In the uplink, base station (BS) receives MW signal from air interface (comb of frequency division multiplexed signals) and converts it in an optical signal. Such a subcarrier multiplexed (SCM) signal in then transmitted to the control station through optical fiber. Therewith, the control station (CS) converts back the SCM signal into the electrical domain, performs the demultiplexing operation, assigns each signal to a channel, and connects it to public switched networks. The downlink works in a similar way. It is also assumed that the double-side bandwidth per carrier is in the range $20-150 \mathrm{MHz}$, to transmit future broad band signals such as 150 or $600 \mathrm{Mbps}$, with the use of modulation schemes with good spectral efficiency, such as QPSK (Quadrature Phase Shift Keying) or 22n QAM (Quadrature Amplitude Modulation).

The main features of the considered approach, with respect to traditional microcellular systems, can be summarized as follows:

1. the BS is simplified, since it does not perform neither modulation/demodulation operations nor control functions (they are indeed achieved in the CS), but it simply executes amplification, frequency 
up/down conversion (usually named RF/IF conversion), automatic gain control (AGC) functions and $\mathrm{E} / \mathrm{O}(\mathrm{O} / \mathrm{E})$ conversion;

2. since the BS is simplified the cost is lowered;

3. the optical feeder gives high flexibility for changes in modulation schemes because it is transparent for all radio signals;

4. the FTTA approach represents an economically viable solution because it could reuse the existing network terminations and the terminals for coax and hybrid fiber coax (HFC) systems.

The efficiency of the operations made in the BS (amplification, E/O conversion etc.) is not critical to assess the feasibility of the present system. Therefore, in this paper, we assume that ideal devices do such operations. In fact, if such non-ideal efficiencies were considered, a further small degradation of the carrier-to-noise ratio would come out. Anyway, this degradation does not represent a real limiting factor for the systems performance as a whole.

The transmission performance of the FTTA system is influenced by several factors. As far as the radio link is concerned, the outage probability, due to the rainfall attenuation, is most important one. While, concerning the optical link several issues impact the performance, such as the effect of the resonant frequency in the laser intensity modulation, the non-linear characteristics of the laser, in particular clipping, and the interplay of laser chirping with fiber dispersion.

The model reported in [12], based on a physical model of the laser dynamics, allows the evaluation of the transmission performance, by taking into account, simultaneously, all the effects influencing the performance.

\section{PERFORMANCE OF FTTA SYSTEMS}

To understand the features and the limits of the FTTA approach in either mobile communication systems or in broadband wireless systems, the following two sections report some relevant results for both the cases.

\subsection{Performance of FTTA systems for mobile communications}

To evaluate the performance transmission of a FTTA system in term of $\mathrm{CNR}$, it has been realized a simulator that generates $\mathrm{N}$ (where $\mathrm{N}$ is the number of RF carriers that modulate the laser) Rayleigh distributed random variables. The generated set of variables is utilized to calculate the CNR, relative to the statistic mean of the optical modulation indices. To obtain results relative to a range of OMI values of almost $40 \mathrm{~dB}$ it is necessary to 
repeat the previous operations a high number of times. Furthermore, the simulator evaluates the CNR for non-faded case, that is, supposing that all the different carriers reaching the antenna have the same optical modulation index. To evaluate the performances of a system affected by multipath fading and optical non-linear distortions, (clipping and static intermodulation), some simulations have been done. The analysis refers, first, to a case in which 3 carriers reach the antenna and the transmission parameter reported in table 1 have been utilized. The average CNR of the central carrier is evaluated, because the carrier in the middle of the frequency band suffers from most severe non-linear distortion [13].

Figure 4 depicts the average CNR versus the average OMI. The results refer to a case in which clipping is not taken into account. This is not a practical case, but this kind of simulation allowed us the comparison between our simulation results and the ones existing in literature [13].

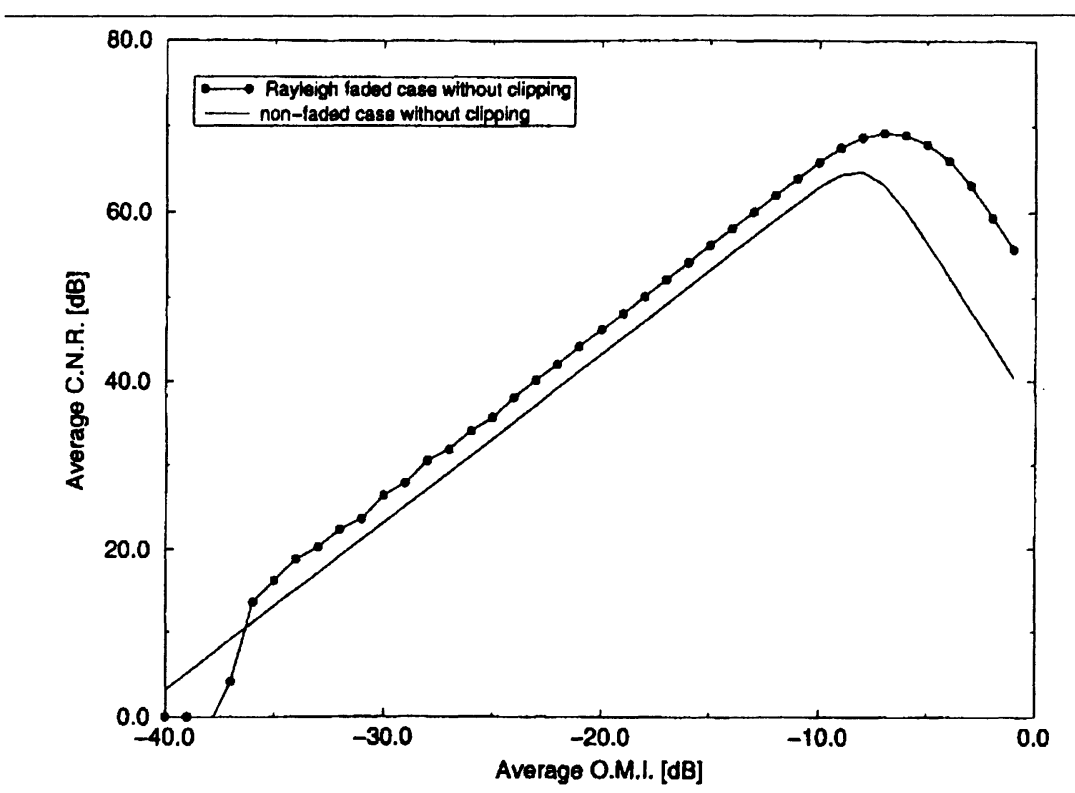

Figure 4. Average CNR versus average OMI taking third-order intermodulation distorsion into account. Comparison between Rayleigh faded and nonfaded case. Three carriers per microcell are considered

In particular in this figure the average CNR both for Rayleigh faded and non faded case is reported. It can be noticed that when the signals suffer from fading, average CNR slightly improves. The reason of this is explained as follows: the third order intermodulation distortion is the product of three different RF signals, and the effects of this are significant only if the signal levels of the three different carriers are simultaneously large, but such a case is rather unusual. 
In figure 5 the comparison between faded cases with and without clipping effects is reported. For low optical modulation indices, the same results are obtained: this means that clipping noise is not dominant and fading multipath increases the average CNR. On the contrary, for high OMI, when clipping noise power becomes dominant, the average CNR of the signal affected by clipping decreases. It is worth noting that clipping occurs when the modulating current exceeds the threshold current: this effect is dominant if just one of the RF carriers has a signal level that can be clipped by the laser.

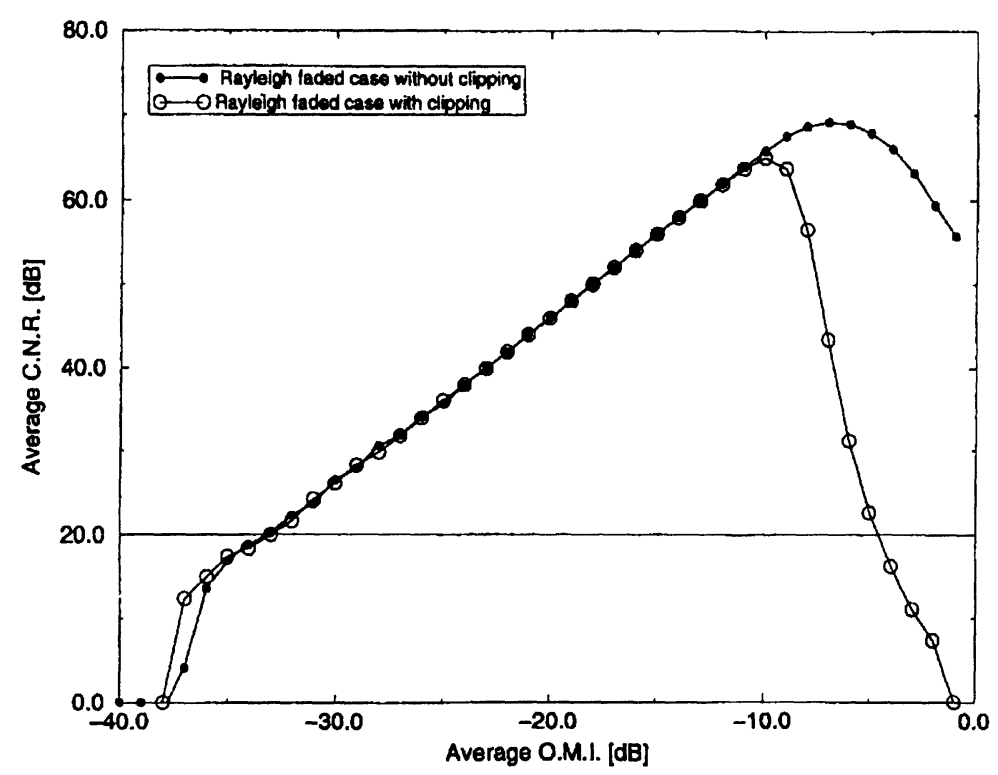

Figure 5. Average CNR versus average OMI. Comparison between Rayleigh faded case with and without clipping. Three carriers per microcell are considered

The simulations are repeated intensifying the number of carriers per microcell. In particular, different cases are considered: 3,5,7,10,13,17 and 20 carriers per microcell. Figure 6 shows the results obtained taking clipping into account and considering the Rayleigh fading. It is worth noting that the maximum OMI that can be employed to obtain a CNR greater than $20 \mathrm{~dB}$ diminishes if the number of carriers increase: this is due to laser clipping effect. Furthermore, as is better shown in figure 7, the maximum value of CNR drops with the growth of the number of carriers. In fact, when the number of carriers that modulate the laser arises, the noise power due to static intermodulation, that is the IM3 power, arises too, and, consequently the carrier to total noise decreases. 


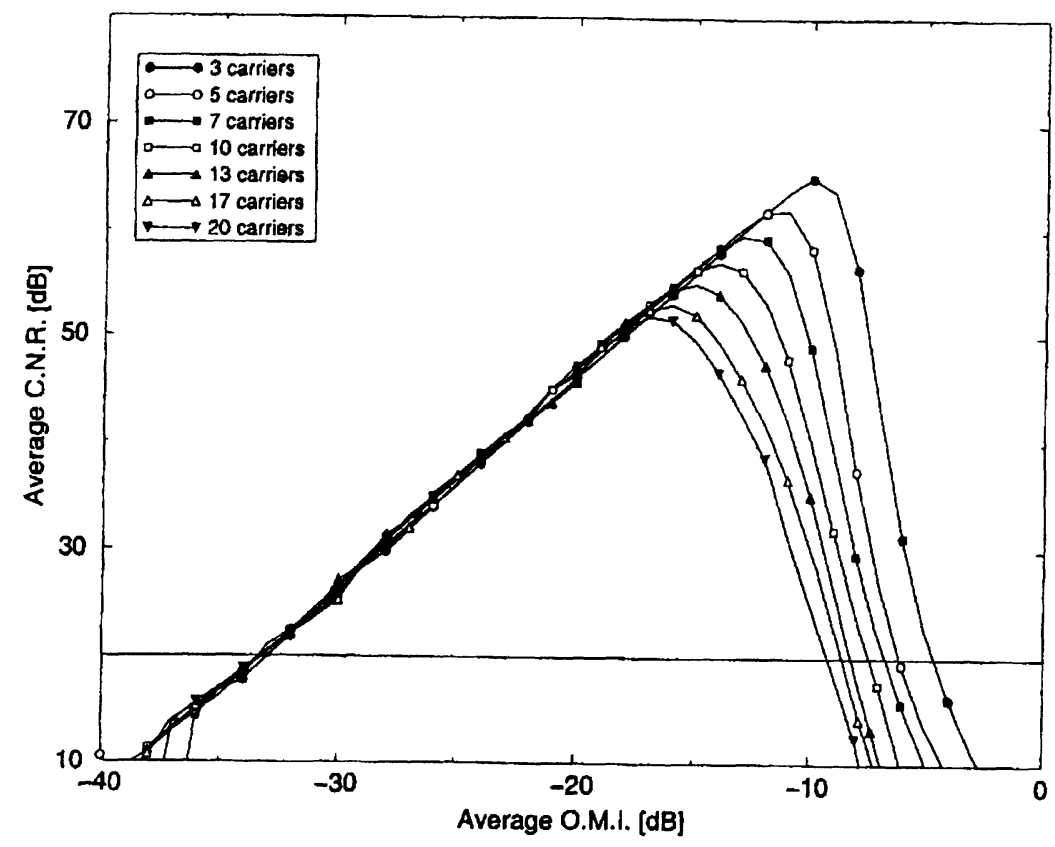

Figure 6. Average CNR versus average OMI for different carrier numbers per microcell

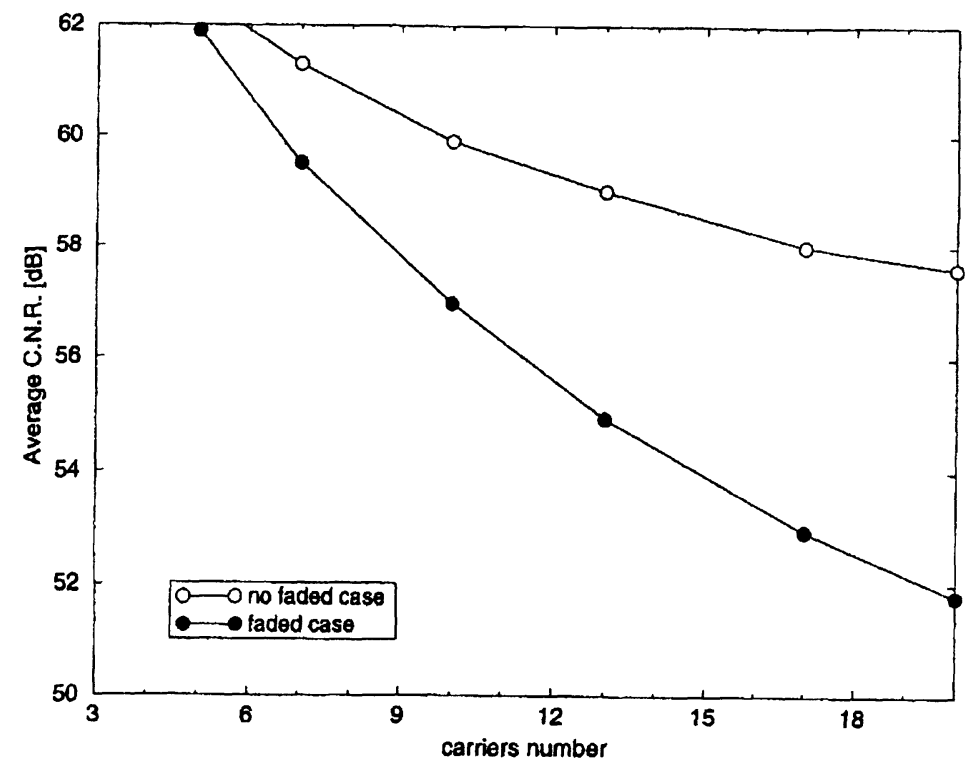

Figure 7. Maximum CNR versus carrier numbers per microcell. Both Rayleigh faded and nonfaded cases are reported 
It has already been underlined that, when RF carriers reach the E/O converter, they have experienced different degrees of propagation loss, multipath fading loss and shadowing loss. This implies that a wide range of power level variation may exist in the received carriers. Now the range of this power level variation is considered, since it has important implication in the dynamic range requirement of an optical fiber link and in particular of the E/O device.

Furthermore, a radio/optical link power budget is reported, realized employing the link parameters reported in table 1. In particular, the maximum and minimum LD input carrier intensity (PO) that can modulate a laser and that allows to obtain a fixed CNR is evaluated. These current values are directly related to the optical modulation index by the expression

$$
m=\sqrt{\frac{2 \cdot P_{i n}}{R_{L D}}} \cdot \frac{\eta_{L D}}{P_{o}}
$$

To study the feasibility of the link, these values are compared with the maximum and minimum RF power that reach the BS antenna. The received power (Pr) can be evaluated using the following expression:

$$
P_{r}=S \cdot P_{t} \cdot\left(\frac{\lambda}{4 \cdot \pi \cdot R}\right)^{2}
$$

where $P_{t}$ is the power transmitted by the mobile station, $\lambda$ is the wavelength of the frequency of interest, $\mathrm{R}$ is the distance between a handset and a base station and $S$ takes into account the shadowing effects $(S=1$ in line-of-sight (L.O.S) propagation). It is assumed that the maximum RF power occurs when a mobile station is placed in L.O.S with the BS station, and it is next to it (this means that the distance between the transmitting and the receiving antennas is about $10-15 \mathrm{~m}$ ). While the minimum power occurs when a mobile station is placed and the edge of the cell (that is $\mathrm{R}$ is equal to the cell radius). In this case the received power is affected by path loss and shadowing. In the calculation, the parameters reported in [14] have been used, where the maximum signal attenuation due to shadowing is set to 30 dB.

The comparison between the maximum and minimum RF received power and the maximum and minimum LD input carrier intensity is necessary to estimate if there is an optimum value of the low noise amplifier (LNA) gain that allows the realization of the link. In particular the radio/optic link is 
feasible, only if the difference between the maximum power values is greater than the difference between the minimum ones. This condition assures that, an optimum LNA gain value, that permits to obtain a CNR greater than the requested one, exists.

Table 1 reports the results obtained in different case, according to the minimum requested CNR ( 15 or $20 \mathrm{~dB}$ in the considered cases); and the microcell radius $(200$ or $300 \mathrm{~m}$ ). To reduce the dynamic range of the received signals, and to guarantee the link accomplishment, different techniques have been proposed: antenna pattern discrimination, automatic power control at the mobile station or macrodiversity. The latter technique, however, that employ the cell splitting concept, don't assure the base station equipment simplification. In fact every BS must be equipped with three $120^{\circ}$ directional antennas and with the adequate electronic devices [15], [4].

\begin{tabular}{|c|c|c|c|c|}
\hline & \multicolumn{2}{|c|}{ Microcell Radius $=200 \mathrm{~m}$} & \multicolumn{2}{|c|}{ Microcell Radius $=300 \mathrm{~m}$} \\
\hline & $\mathrm{CNR}=15 \mathrm{~dB}$ & $\mathrm{CNR}=20 \mathrm{~dB}$ & $\mathrm{CNR}=15 \mathrm{~dB}$ & $\mathrm{CNR}=20 \mathrm{~dB}$ \\
\hline 3carriers & Possible with & Possible with & Possible with & Possible with \\
\hline & $\mathrm{G}=21.11 \mathrm{~dB}$ & $\mathrm{G}=25.51 \mathrm{~dB}$ & $\mathrm{G}=24.63 \mathrm{~dB}$ & $\mathrm{G}=29.03 \mathrm{~dB}$ \\
\hline 5 carriers & Possible with & Possible with & Possible with & Not possible \\
\hline & $\mathrm{G}=20.31 \mathrm{~dB}$ & $\mathrm{G}=25.11 \mathrm{~dB}$ & $\mathrm{G}=23.83 \mathrm{~dB}$ & \\
\hline 7 carriers & Possible with & Possible with & Possible with & Not possible \\
\hline & $\mathrm{G}=19.91 \mathrm{~dB}$ & $\mathrm{G}=25.01 \mathrm{~dB}$ & $\mathrm{G}=23.43 \mathrm{~dB}$ & \\
\hline & Possible with & Not possible & Possible with & Not possible \\
\hline $\begin{array}{l}13 \\
\text { carriers }\end{array}$ & $\begin{array}{l}\text { Possible with } \\
G=19.51 \mathrm{~dB}\end{array}$ & Not possible & Not possible & Not possible \\
\hline $\begin{array}{l}17 \\
\text { carriers }\end{array}$ & $\begin{array}{l}\text { Possible with } \\
G=19.49 \mathrm{~dB}\end{array}$ & Not possible & Not possible & Not possible \\
\hline $\begin{array}{l}20 \\
\text { carriers }\end{array}$ & $\begin{array}{l}\text { Possible with } \\
\mathrm{G}=18.89 \mathrm{~dB}\end{array}$ & Not possible & Not possible & Not possible \\
\hline
\end{tabular}

TABLE 1 Microcell Link Power Budget

\subsection{Performance of FTTA systems for wireless broadband communications}

The performance of the optical link has been reported in terms of the CNR versus the optical modulation index (OMI) of the single channel. It is worth noting that such an analysis is similar to the one we reported in [16]. However, the total bandwidth, in the cases treated in the present paper, is quite different than in the case of CATV signal distribution: we consider 
here, in some cases, a total bandwidth of the order of the $\mathrm{GHz}$, that is quite larger than the one considered for CATV systems. In fact, we expect a greater impact of the chirping/dispersion impairment.

In fact, figures 8 - 10 show the transmission performance of the system, in terms of the CNR versus the optical modulation index (OMI) of the single channel, varying several system parameters.

A general observation of all those figures, which is worth remarking, is that the increasing of the signal power, directly related with the OMI, produce an increasing of the CNR, until non-linear distortions become significant, so causing an increasing of the NLD contribution to the noise power. This explains why there is an optimum value of the OMI, which allows the maximum CNR to be reached.

The impact of the number of SCM carriers on the performance is depicted in figure 8 , where the performance are reported for different values of the number of carriers, assuming a double-side bandwidth of $150 \mathrm{MHz}$ for any single channel. In fact, it can be noticed that the CNR decreases with the increasing of the number of carriers. Basically, two reasons can be individuated for this: i) the overall bandwidth of the SCM signal increases and, consequently, the noise contributions grow accordingly; ii) the probability of clipping events (e.g. the clipping induced total distortion) increases when there are more carriers [16].

To investigate the impact of the single channel bandwidth on the system performance, figure 9 shows the CNR versus the OMI for three different values of the channel bandwidth, assuming the transmission of $20 \mathrm{SCM}$ channels. It is evident as the increasing of the bandwidth leads to an increasing of the noise contributions. Moreover, the increasing of the bandwidth, as a whole, could generate further distortion contributions due to both chirping/dispersion and the effect of the resonance frequency of the laser. 


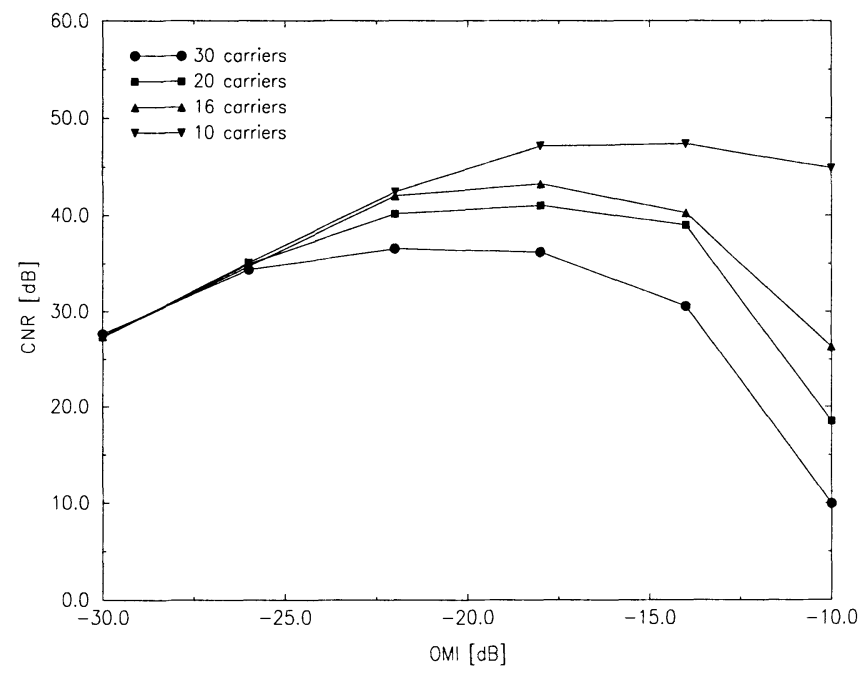

Figure 8. CNR versus the OMI, for different numbers of carriers; the RF comb starts from $200 \mathrm{MHz}$

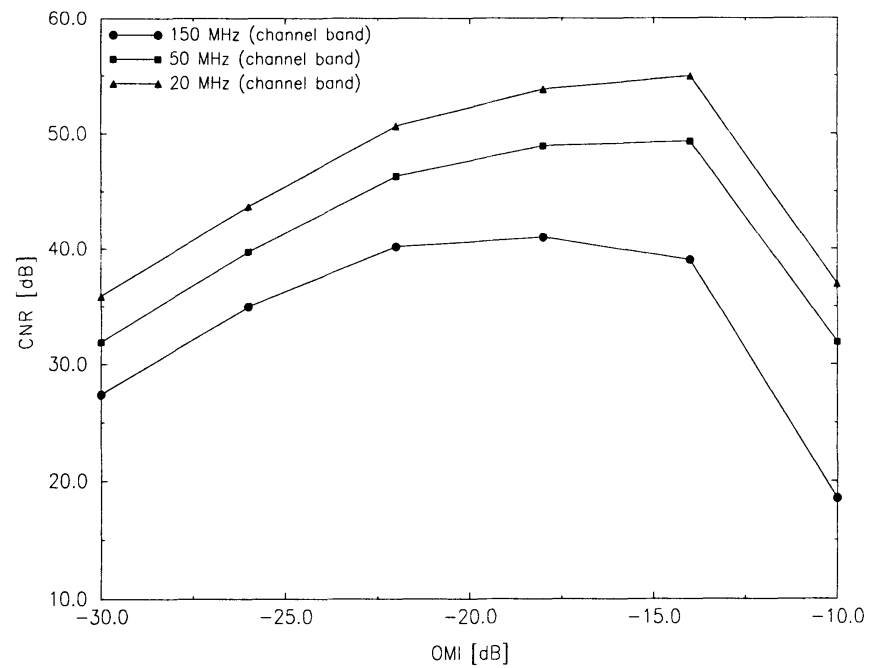

Figure 9. CNR versus the OMI, for different values of the channel bandwidth; the RF comb starts from $200 \mathrm{MHz}$

In order to evaluate the effect of source chirping (in commingling with fiber dispersion) and of the resonance frequency of the laser on the system 
performance, it is useful to observe figure 10. Here it is assumed to transmit 20 carriers. It is also presumed a fiber optic link length of $30 \mathrm{~km}$. Such a feeder length is realistic, if we consider that a control station links a certain number of base stations located in a geographical area. The MW signal, consisting of channels' comb, is received from the antenna, and is down shifted near the baseband (200 MHz) in one case, and to $1 \mathrm{GHz}$ in the other case. In both the cases the performance are evaluated either considering the chirping effect or not.

By comparing the curves relating to the comb initial frequencies of 200 $\mathrm{MHz}$ and $1 \mathrm{GHz}$, respectively, it is possible to observe a difference of few $\mathrm{dB}$ ( 3 in the worst case). This is due to the fact that, when the laser operates close to the resonance frequency (the relaxation oscillation frequency), further distortions are introduced. Therefore, the channels' comb has to be placed in order to avoid this effect.

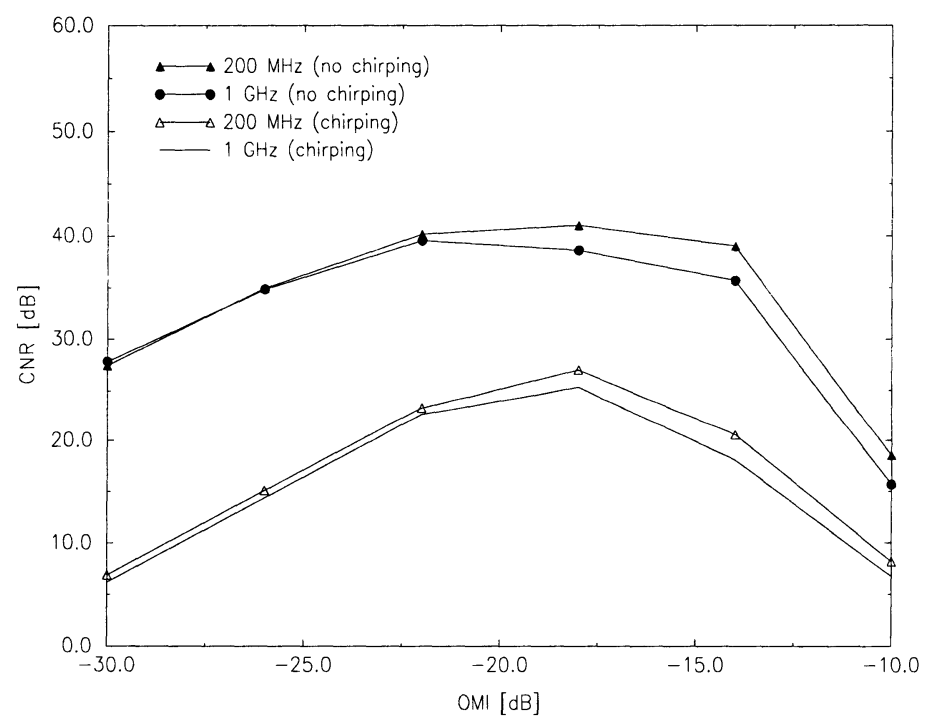

Figure 10. CNR versus the OMI, for different values of the start frequency and showing the chirping effect

As far as the system design is concerned, it is useful to derive some qualitative considerations from the obtained results. For instance, since the required CNR values, which allow an error probability of 10-6 to be reached, for QPSK and for 16 QAM modulation schemes, are 13.8 and $18.8 \mathrm{~dB}$, respectively, we could say that the considered system can transmit up to 30 carriers, each one having a double-side bandwidth of $150 \mathrm{MHz}$. On the other 
hand, if the 64 QAM modulation scheme is desired, the required CNR value of $34.8 \mathrm{~dB}$ pose restrictions on the number of carriers, and requires a limited range of value of the OMI (with 20 carriers the OMI should be in the range $26 \div-13 \mathrm{~dB}$ ). This means that QPSK or 64 QAM modulations can be favorably adopted for this application.

It is also to be highlighted that the impact of chirping effect is absolutely not negligible since, in case of long fiber optic link in the trunk (e.g. $30 \mathrm{~km}$ ), it brings about a penalty of more than $13 \mathrm{~dB}$, on the CNR value.

Again, considering the required values of CNR for QPSK and 16 QAM modulation schemes, we could say that, in this case, just a limited range of values for the OMI is admissible, considering a transmission of 20 carriers of $150 \mathrm{MHz}$ bandwidth each.

\section{DISCUSSION AND CONCLUSIONS}

The application of the hybrid approach in either mobile communication systems or in broadband wireless systems can be successful.

In the analysis of mobile systems, both Rayleigh multipath fading and optical non-linear distortions were taken into account. The results, obtained with several carriers transmitted from a single source, show that, for high optical modulation indices, clipping effect, more than static intermodulations effect, diminishes the average CNR at the photodetector receiver. Therefore, the number of carriers allowed to be used per microcell is drastically limited by clipping noise: to assure a good service quality it is indispensable to regulate the number of carriers per microcell or employ adequate technique to reduce the dynamic range of the received signals.

As far as the wireless broadband systems, the following considerations can be made. The rainfall attenuation is the basic limitation of the performance of the radio link: it introduces an outage probability. A typical and relevant example of FTTA system has been presented and a performance analysis has been achieved. In fact, in the case of small cell radius $(300 \mathrm{~m})$ the outage probability was evaluated to be acceptable, while in the case of large cell radius $(1 \mathrm{~km})$ the outage probability was too large for the considered applications.

As far as the optical link is concerned, noise contributions, non-linear distortions and the combined effect of chirping/dispersion are the most limiting factors. In particular, optical non-linear distortions are basically due to the threshold characteristic of the laser, which gives rise to clipping effect. Nevertheless, other non-linear contributions, such as the non-linearity of the laser characteristic even above threshold or the saturation effect, are 
intrinsically taken into account into the physical model used in the present analysis.

The analysis revealed that this kind of system allows the transmission of some tens of broadband carriers, using two relevant modulation schemes: QPSK and 16 QAM.

In general, it is possible to say that a tradeoff exists between the number of carriers and the channel bandwidth. Furthermore, the impact of chirping could be significant.

As a result, the analysis reported in this paper assesses the feasibility of the FTTA approach for realizing broadband services distribution systems. In the design of this type of systems it is necessary to consider the following points.

1. Dimensioning of the radio link, in order to obtain a fixed outage probability.

2. In practical systems automatic gain control circuits are required in the base station to compensate for the rainfall attenuation.

3. The optical link has to be designed taking into account non-linear distortions contribution arising by direct modulation of a single mode laser source, and the chirping/dispersion contribution, especially if the feeder length is some tens of kilometers long.

\section{ACKNOWLEDGEMENTS}

I wish to thank my student Alessandra Rohr, for her help in preparing this paper.

\section{BIBLIOGRAPHY}

[1] Special Issue on Fiber-Optic Microcellular Radio Communication System and Their Technologies, IEICE Trans. on Comm., vol. E76, no. 9, pp. 1059-1185, 1993.

[2] K. Morita, H. Ohtsuka, "The new generation of wireless communications based on fiberradio technologies", IEICE Trans. on Comm., vol. E76, no. 9, pp. 1061-1068, 1993.

[3] J. Namiki, et al., "Optical feeder basic system design for microcellular mobile radio", IEICE Trans. on Comm., vol. E76, no. 9, pp. 1069-1077, 1993.

[4] W.I. Way, "Optical fiber-based microcellular systems: an overview", IEICE Trans. on Comm., vol. E76, no. 9, pp. 1091-1102, 1993.

[5] C.J.Chung, I.Jacobs, "Simulation of the effects of laser clipping on the performance of AM-SCM ligthwave systems", IEEE Photon.Techn.Lett., Vol.3, Nº11, pp.1034-1036, 1992.

[6] R.Sabella," Performance evaluation of optical fiber link in microcellular mobile communications systems ,"Microwave and Optical Technology Letters, Vol. 18, no 3, June 1998. 
[7] H. Ogawa, " Microwave and millimiter-wave fiber optic technologies for subcarrier trasmission systems," IEICE Trans. Electron., vol. E76-B, no 9, pp. 1078-1090, 1993

[8] R. Heidemann et al., " Demonstrating the enabling technology for broadband wireless local loop: ACTS FRANS and other hybrid fiber radio trials," Conf. Proc. ISSLS '98, Venice, Italy, pp. 401-407.

[9] J. J. O'Reilly et al., " Optical generation of very narrow linewidth millimeter wave signals," Electron. Lett., vol. 28, pp. 2309, 1992.

[10] M. Varasi et al., " Velocity matched modulators for wide bandwidth, high efficiency, and high extinction ratio," presented at the FOTONICA '99, Trento, Italy, June 2-4, 1999.

[11] H. Harada, H. J. Lee, S. Komaki, and N. Morinaga, " Performance analysis of fiber-optic millimiter-wave band radio subscriber loop," IEICE Trans. Electron., vol. E76-B, no 9, pp. 1128-1135, 1993.

[12] R. Sabella, "Performance Analysis of Wireless Broadband Systems employing optical fiber links," IEEE Trans. Commun.Vol. 47, no.5, May 1999.

[13] H. Mizuguti, T. Okuno, S. Komaki, N. Morinaga "Performance Analysis of fiber link for microcellular mobile communication system”, IEICE Trans. Electron. Vol. E76-C, No 2, 271-278, 1993.

[14] O. K. Tonguz et al., " Personal Communications Access Networks Using Subcarrier Multiplexed Optical Links," J. Lightwave Techìnol.,Vol. 14, June 1996, pp. 1400-1409.

[15] T. S. Chu and M. J. Gans, " Fiber Optic Microcellular Radio," IEEE Trans. Veh. Technol., Vol. 40, No. 3, 1991, pp. 599-606.

[16] R. Sabella, M. Paciotti, and A. Di Fonzo, " Analysis of clipping effects on AM-VSB CATV subcarrier multiplexed optical systems," J. Opt. Commun., vol 19, pp. 72-74, Apr. 1998.

Roberto Sabella was born in Roma, Italy, in December $10^{\text {th }} 1962$. He received the degree in electronics engineering (Laurea in Ingegneria Elettronica) from the University "La Sapienza" of Roma, Italy, in 1987. He then joined Ericsson Telecomunicazioni, Italy, where he was a Hardware Designer for about one-and-ahalf years, and then as a Researcher on advanced fiber-optic communications. His research interests are in high-speed optical communication systems and optical networks. Since 1991, he led a group working on the modeling and simulation of advanced communication systems. He holds two patents on optical cross-connects, is co-author of a book on high-speed optical communications and author of more than 70 papers on scientific/technical journals and international conferences. Since May 1997, he has been with CoRiTel consortium as research technical co-ordinator. Since 1999, he is the research manager of the Ericsson research unit in Roma.

He has been Lecturer (professore a contratto) at the University of Rome "Tor Vergata" and at the Polytechnic of Bari, Italy. Mr. Sabella is member of the IEEE/LEOS Technical Committee on Optical Networks and Systems, and member of the editorial board of the new international journal Photonic Network Communications (New York: Kluwer Academic). For the same journal he has operated as guest editor for the special issues on WDM transport networks. Moreover, he has been one of the guest editors of a special issue on optical networks, for the journal Computer Networks (Elsevier).

$\mathrm{He}$ is also a guest editor for the feature topic "Optical Networking Solutions for Next Generation Internet Networks", to appear on the IEEE Communications Magazines. 